mountable without new approaches.

In his review of the modulation theory of cosmic rays, L.A. Fisk describes the present situation as "in a state of wary optimism". Although this may indeed be the case in certain areas, it remains to be seen how many of the ideas will survive another decade. It is useful to note in this connection that one of the most important events in solar system plasma physics in the last few years was the finding of a very interesting electric potential structure above the aurora (described in Vol. III, 1.5 and 1.6). The possibility of the presence of an electric field along magnetic field lines in a collisionless plasma should receive wide attention by astrophysicists in general. In this connection it may be recalled that various exotic acceleration mechanisms of energetic particles have been described in the past by assuming that such a parallel electric field cannot exist. This unexpected finding of the potential structure has already considerably changed the course of our thinking and will continue to do so during the next decade. New findings by deep space probes may also drastically alter our ideas on the heliosphere. In this respect, the authors could have been a little more bold by injecting new ideas as it is rather difficult to do so in a paper for a regular journal these days, given the conservative attitude of many referees.

As stressed earlier, however, there is no other up-to-date and comprehensive review available which covers the entire scope of solar system plasma physics. It is hoped that the book will be widely read by everyone who is interested in plasma physics in general.

S.-1. Akasofu is Professor of Geophysics at the Geophysical Institute of the University of Alaska, Fairbanks, Alaska.

\section{Photoresearch for physical chemists}

\section{D.W. Turner}

Photoabsorption, Photoionization and Photoelectron Spectroscopy. By J. Berkowitz. Pp. 469. (Academic: New York and London, 1979.) $\$ 61, £ 39.80$.

THE story of the exploration of that area of the electromagnetic spectrum which falls between the optical and the X-ray regions is an extremely exciting one, partly in terms of the succession of new theoretical and interpretative problems that have arisen, but most especially because of the elegant, painstaking and sometimes heroic experimental techniques which have been called for. From the earliest pioneering work of Schumann and Lyman to the present day, advance has always been associated with new techniques in the hands of gifted experimenters. Some major leaps forward and acceleration in activity in the area can be identified in retrospect the observation of molecular Rydberg series in the 1930s, the application of photoionization threshold measurements in the 1950s and photoelectron spectroscopy in the following decade. The latter gave, for the first time, access to the inner valence shells of complex molecules and at this stage a unification with parallel developments in the soft $X$-ray region established an area of work which had become essentially chemical in its applications, though the methods were those of the physics laboratory. The present text is the first which has made a serious attempt to draw all of these threads together and show the application of these and other related techniques to the understanding of the structure of some quite complex molecules and their ions.

After a survey of some fundamental principles and an outline of physical processes underlying the absorption of high-energy radiation and ionization processes generally, the author devotes three chapters to describing photo- absorption below the first ionization limit, quasi-discrete states above the first ionization limit and the physics of the ionization continua. There then follows a discussion of photoabsorption and photoionization cross-sections for selected molecules in which all the information available for each case is brought together for critical evaluation, and then a similar critical discussion of partial cross-sections of a range of molecules in which extensive use is made of photoelectron spectral data. A separate chapter considers the problem of the angular distribution of photoelectrons.

This is essentially theoretical with a rather small number of applications to simple molecules being described. The book ends with a survey on instruments and methods which is particularly well done.

This is a text produced to an extremely high standard and the author speaks with authority and clarity. It is to be recommended particularly to research workers in the field. Chemical physicists generally will find it a valuable reference text.

D. W. Turner is Reader in Physical Chemistry at the University of Oxford, UK.

\title{
Principles of gel permeation
}

\section{J.V. Dawkins}

Modern Size-exclusion Liquid
Chromatography: Practice of Gel Permeation and Gel Filtration Chromatography. By W.W. Yau, J.J. Kirkland and D.D. Bly. Pp.476. (Wiley: New York and Chichester, UK, 1979.) $\$ 36.60, £ 17.45$.

STUdiEs of the chromatographic fractionation of macromolecules by a sizeexclusion mechanism have progressed along two parallel paths. Gel filtration, which is widely used for analytical and preparative separations of biological macromolecules in aqueous media, resulted from the preparation of soft porous gels, as described by Porath and Flodin in Nature $(183,1657-1659 ; 1959)$. Rigid porous packings such as crosslinked polystyrene, silica and glass particles for fractionations of synthetic polymers in crganic media by the technique known as gel permeation chromatography were reported in 1964-1967. The theme bringing the two techniques together in this book is the use of rigid microparticulate packings for performing fast (minutes) highresolution separations. The decrease in the separation time from several hours in the classical size exclusion techniques has followed from the amazing advances, to which Kirkland has made important contributions, that have occurred in liquid chromatography during the past decade. Whilst high-speed separations of many synthetic polymers may be performed with porous cross-linked polystyrene microspheres, suitable microspherical rigid packings for widespread routine use in gel filtration are still in an active state of development.

Following a brief introductory chapter, there are three chapters on retention mechanisms, band-broadening and resolution. The authors have concentrated on principles rather than detailed theoretical treatments, so the practitioner can clearly identify the important variables which determine separation power and column performance. Chapters 5-11 are the most valuable sections of the book with comprehensive accounts of equipment and detectors, column packings and technology, operating variables, laboratory techniques, calibration, data handling and special techniques. The experimental aspects are well illustrated with figures and detailed tables, with more examples taken from gel permeation than from gel filtration, but the bibliography is very selective with the total number of cited references for these seven chapters below 200. Thus, it is surprising that the 